\title{
Translational Aspects of Blood-Brain Barrier Transport and Central Nervous System Effects of Drugs: From Discovery to Patients
}

\author{
ECM de Lange ${ }^{1}$ and M Hammarlund-Udenaes ${ }^{2}$
}

\begin{abstract}
The development of CNS drugs is associated with high failure rates. It is postulated that too much focus has been put on BBB permeability and too little on understanding BBB transport, which is the main limiting factor in drug delivery to the brain. An integrated approach to collecting, understanding, and handling pharmacokinetic-pharmacodynamic information from early discovery stages to the clinic is therefore recommended in order to improve translation to human drug treatment.
\end{abstract}

\section{INTRODUCTION}

Drug development for central nervous system (CNS) diseases has encountered serious problems with only $9 \%$ of compounds that entered Phase 1 studies surviving to launch. Over 50\% of this attrition is the result of failure to demonstrate efficacy in Phase 2 studies. Compounds that worked effectively on cloned human proteins and in animal models have been found ineffective in human disease. ${ }^{1}$ Reasons for the failure of these CNS drug candidates can, at least in part, be found in inconclusive pharmacokinetic data. For example, unbound (free) drug concentration data, particularly regarding blood-brain barrier $(\mathrm{BBB})$ transport and intrabrain distribution may not have been used. Inconclusive pharmacodynamic data and variability in the available data as a result of the heterogeneous nature of CNS pathologies in humans can also cause problems. ${ }^{2,3}$

Clearly, CNS drug research so far has not yet provided solutions and the question remains: how to place the right drug, at the right time, at the right concentration, in the right place? To answer this question, it is necessary to ask several more. How can we obtain information on (what can be referred to as) the site of action in the CNS? How can we appropriately diagnose CNS diseases, especially in the early stages? How can we accurately determine the effects of the drug treatment on the disease? We need better insight into the fates and associated effects of CNS drugs in the brain to develop better treatments. We need to consider the multiple factors that govern the pharmacokinetics of these drugs at their sites of action in the CNS and we need to integrate all of them into a general framework that can be used to predict human CNS target site pharmacokinetics. ${ }^{4}$
The BBB is the main obstacle preventing drugs in the blood stream from entering the brain. It is an organ that, in essence, is present to help the brain work properly. This function is governed by the selective transport of nutrients and other essential substances needed by the brain in an inward direction, and the selective transport of the waste products of brain function in an outward direction. The selectivity is maintained by various transport functions of the otherwise very tight membrane. Efflux transporters also protect the brain from unwanted chemical influence, with $\mathrm{P}$-glycoprotein (P-gp) as the best-known example.

Our understanding of the BBB has improved significantly over recent years, in particular with respect to the molecular mechanisms that govern the tightness of the $\mathrm{BBB}$ and the various functions of the individual parts of the neurovascular unit, of which the BBB (i.e., the endothelial cells of the capillary walls in the brain) is one..$^{5}$ The other functional parts of the neurovascular unit include the astrocytes, the pericytes and the basal membrane (Figure 1). This intricate system has to be conquered and outwitted by pharmaceutical scientists to develop treatments for CNS diseases.

One of the significant steps in improving our understanding of drug transport across the $\mathrm{BBB}$ was the separation of the rate of transport across the $\mathrm{BBB}$ from the extent of transport across the $\mathrm{BBB}$, and the identification of unbound drug as the important entity. ${ }^{6}$ This latter point also included separating drug binding in the brain and blood from the processes of $\mathrm{BBB}$ transport. Further progress is required in developing new routines within drug industry discovery/development settings, although the

\footnotetext{
${ }^{1}$ Leiden Academic Centre for Drug Research, Division of Pharmacology, Leiden University, Gorlaeus Laboratories, Leiden, The Netherlands; ${ }^{2}$ Department of Pharmaceutical Biosciences, Uppsala University, Uppsala, Sweden. Correspondence: M Hammarlund-Udenaes (mhu@farmbio.uu.se) 


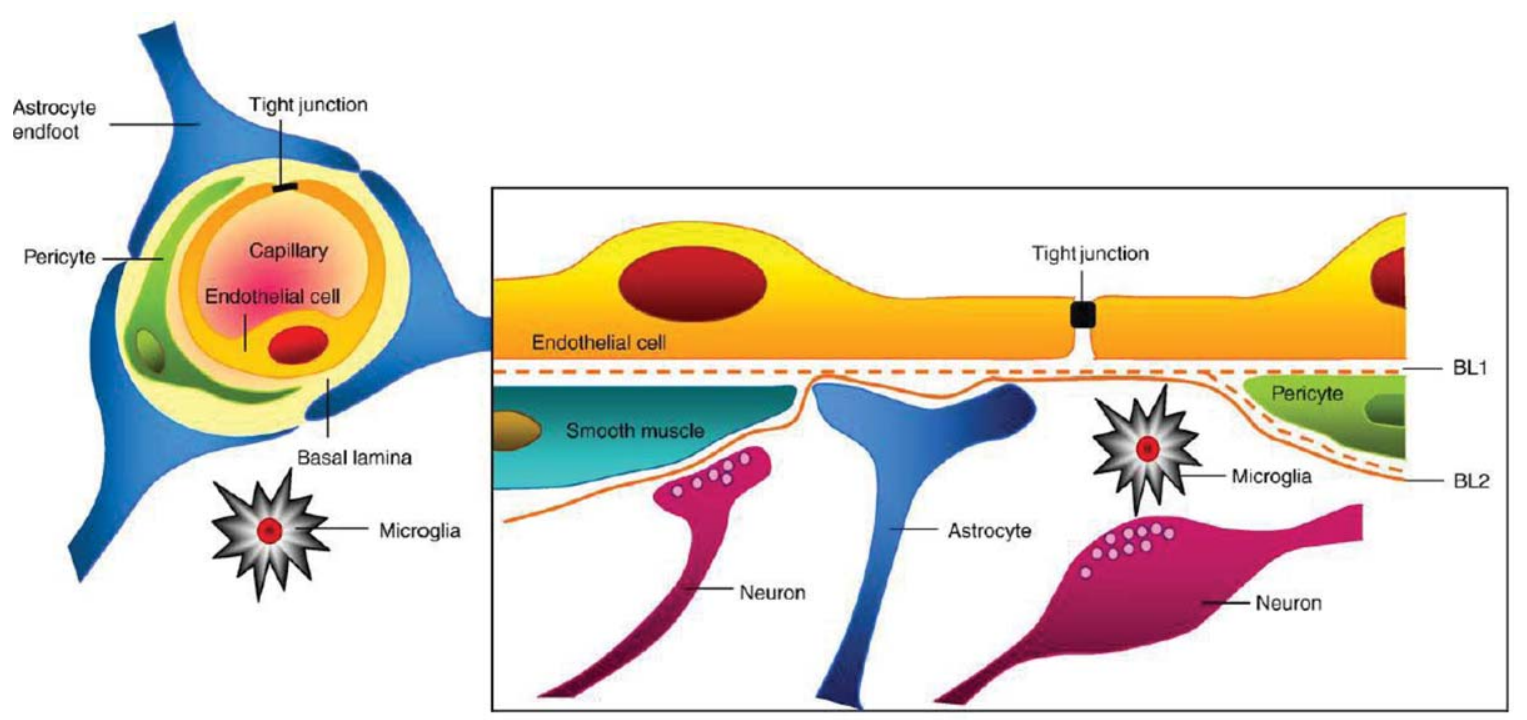

Figure 1 The neurovascular unit. The blood-brain barrier is made up of the endothelial cell layer. From Abbott, Neurobiol of Disease 2010 with permission from Elsevier.

principles are catching on. New methods of addressing these issues are presented in this review.

Once it has passed the $\mathrm{BBB}$, the drug can reside in brain extracellular fluid (ECF), brain cells and their different cellular compartments, or the cerebrospinal fluid (CSF). ${ }^{4}$ The bloodCSF barrier (BCSFB) in the choroid plexus also needs to be taken into consideration, as it is a separate barrier to the movement of drugs into the CSF. ${ }^{7}$ Transport of the drug between the different parts is governed by diffusion, binding, and fluid flow mechanics, but also by active transport mechanisms at the level of the brain parenchymal cellular compartments such as astrocytes, microglia, and neurons. ${ }^{8}$ Metabolism and degradation of the drug in the brain may also influence its local concentrations. ${ }^{9,10}$

The rate and extent of BBB transport and intrabrain distribution of a drug can also depend on species, ${ }^{11}$ age and gender, ${ }^{12}$ presence of disease, ${ }^{13,14}$ diet, ${ }^{15}$ and time of day. ${ }^{16}$ Disease mechanisms and the influence of disease on the BBB and the neurovascular unit are currently under investigation in many laboratories and some progress has been made. However, we still know little about how these disease states influence drug transport to the brain. ${ }^{14,17,18}$

Pharmacometric models allow the contributions and variability of the individual processes of drug administration, CNS drug distribution and ultimately pharmacological effect to be parameterized and further understood. Differences between conditions can be revealed by separating drug properties from biological system characteristics. Physiologically based pharmacokinetic (PBPK) models have applied this separation for decades, although time resolution and the unbound drug concentrations have not been specifically taken into account. ${ }^{1,20}$ Strategic, systematic CNS drug research based on the "Mastermind Approach," a term introduced to indicate the inclusion of mathematical modeling using unbound drug concentrations, time resolution, and advanced preclinical experimental designs, ${ }^{4}$ will result in informa- tion on the individual processes on the causal path between drug dosing and CNS effect in animals, that can be translated to the human situation.

In this review, we discuss new findings regarding the principles, methods, and results of studies designed to improve the assessment of the BBB in the evaluation of new drugs for CNS diseases. The basis of the study is translational, and the purpose is to review the information to improve CNS drug treatment in humans.

\section{PROCESSES THAT GOVERN THE LOCAL PHARMACOKINETICS OF UNBOUND (FREE) DRUG IN THE CNS}

\section{Entering and leaving the brain}

Drug transport into, within, and out of the brain is governed by the free unbound drug concentrations in plasma and by transport across the brain barriers, both the BBB and the BCSFB. Other factors include ECF bulk flow and CSF turnover, extracellularintracellular exchange, brain tissue binding and brain drug metabolism. It is important to note that transport across the blood-brain barriers can occur by simple diffusion, facilitated diffusion, vesicle transport, active transport, or combinations of these, depending on the drug. All these processes occur concomitantly, and each will influence the rate and extent of transport of the others, such that the interrelationships need to be considered to predict CNS target site concentrations, and the resulting drug effects. ${ }^{4,6}$

As many of the important known CNS targets are membrane-bound receptors facing the brain ECF, it is important to know the concentrations in this compartment to predict the availability of the drug for interaction with its CNS target. The drug properties and pharmacokinetic processes that govern drug concentrations at specific CNS sites must be understood, ideally with measurements of target engagement and drug effects. ${ }^{3}$ 


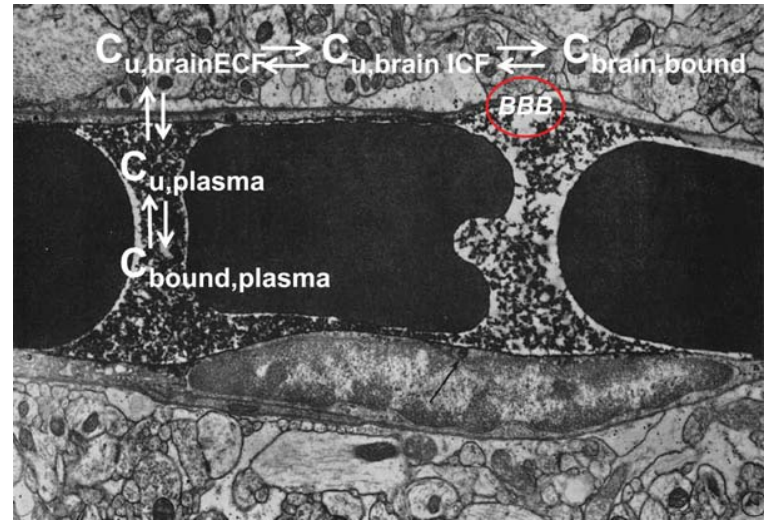

Figure 2 Schematic drawing of the transport of drugs between the brain capillaries across the blood-brain barrier (BBB) to brain parenchyma, including the equilibria between unbound- and bound-drug concentrations (C) in the different compartments. ECF refers to the extracellular fluid except plasma; ICF refers to the intracellular fluid. Most of the drug is assumed to be bound intracellularly rather than to in the interstitial membranes. The drawing is made on an electron micrograph picture from Reese and Karnovsky. ${ }^{84}$ The three black cells are red blood cells. The picture is published with permission from ref. 84 .

The main entry point of drug molecules into the brain is, despite its tight junctions and active efflux transporters, through the $\mathrm{BBB}$. The $\mathrm{BBB}$ is also the main exit point. The brain can, therefore, from a pharmacokinetic perspective, be seen as an advanced distribution compartment. The physiological processes for drug transport into the brain include diffusion through the brain endothelial cells, which requires a certain degree of lipophilicity; active uptake by transporters at the luminal membrane; and/or transcytosis which can be either adsorptive or receptormediated. The processes of drug molecular movement and equilibration are further described in Figure 2.

Paracellular transport is of minor importance due to the tight junctions, except for small hydrophilic drugs, which do not diffuse easily across the brain endothelial cell membranes. Active efflux by P-gp, breast cancer resistant protein (BCRP), and other transporters hinders the (net) influx and thereby counteracts the extent of uptake into the brain, while active uptake into the brain improves uptake and counteracts elimination. Passive fluid flow from the brain parenchyma to the CSF is probably less important for lipophilic compounds, but more important for the elimination of hydrophilic compounds with low passive permeability properties at the BBB. Metabolism within the endothelial cells is a possible protecting mechanism, but little is known still about the quantitative importance of this process. ${ }^{21}$

The pharmacokinetic principles describing drug transport to and from the brain involve the rate and extent of transport. ${ }^{6}$ The rate of transport can be measured as the in vivo permeability surface area product across the $\mathrm{BBB}$, measured using methods like the in situ brain perfusion technique. ${ }^{22}$ In vitro cell-based methods also give information on the rate, although this is not necessarily BBB-specific. For drugs where a rapid effect is wanted, this is an important parameter to study. In cases where drugs are intended for repeated daily dosing to treat chronic diseases the extent of transport is much more important. The extent of transport can be studied using several methods, as described below. ${ }^{23}$ If a drug is permeable enough at the intestinal mucosa to be administered orally, there seems to be no problem with the BBB transport, at least from a rate perspective.

The steady-state extent of transport across the $\mathrm{BBB}$ is driven by the relative capacity of passive transport, active uptake and active efflux at the $\mathrm{BBB}$. The ratio of unbound-drug concentrations in brain ECF to those in plasma, $\mathrm{K}_{\mathrm{p} \text {,uu,brain }}$, can be used to describe this BBB equilibrium (Eq. 1). ${ }^{6,24}$

$$
K_{p, u u, \text { brain }}=\frac{A U C_{u, \text { brain }}}{A U C_{u, \text { plasma }}}=\frac{C L_{\text {in }}}{C L_{\text {out }}}
$$

where AUC describes the area under the concentration-time curve of unbound drug in brain and plasma, and $\mathrm{CL}_{\mathrm{in}}$ and $\mathrm{CL}_{\text {out }}$ describe the net clearances (permeability surface area products in $\mu^{*} \min ^{-1 *} \mathrm{~g}_{\text {b brain }}{ }^{-1}$ ) into and out of the brain, respectively. The AUC expressions after a single dose can be replaced by steady-state concentrations. Eq. 1, therefore, describes the relationship between the rates of transport and the extent of equilibration.

A dominance of passive transport in both directions gives a ratio of unity. Other values indicate active transport either into or out of the brain. If $\mathrm{K}_{\mathrm{p} \text {,uu,brain }}$ is below unity, this indicates a more dominating active efflux, while a $\mathrm{K}_{\mathrm{p} \text {,uu,brain }}$ above unity shows that the drug has more active influx than efflux. Thus, the efficiency of the net active transport for a certain drug will have a

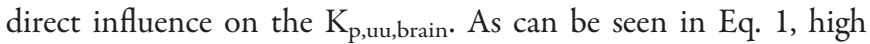
clearance into and out of the brain will give the same steady-state relationship between brain and plasma concentrations as low clearance into and out of the brain. This is the main reason why the permeability per se is relatively unimportant for the extent of transport to the brain and thereby also for the pharmacological effects during repeated dosing.

Calculations using the total concentration ratio $\mathrm{K}_{\mathrm{p} \text {,brain }}$ (or $\log \mathrm{BB}$ ) are still being used by some drug companies and academic institutions to estimate BBB transport, which has severely hampered the industry's ability to choose suitable compounds for CNS action. ${ }^{6,25,26}$ In the pharmaceutical industry, a value for $\mathrm{K}_{\mathrm{p} \text {,brain }}$ of $>2(\operatorname{logBB}>0.3)$ was arbitrarily chosen, without a sound scientific basis, as a cut-off point for selecting useful CNS drugs. $\mathrm{K}_{\mathrm{p} \text {,brain }}$ includes binding in brain and the protein binding in plasma, apart from the BBB equilibrium (Eq. 2). This obscures both the net $\mathrm{BBB}$ transport itself, and also the evaluation of whether the drug is a successful candidate for CNS action.

$$
K_{p, \text { brain }}=\frac{A U C_{\text {tot }, \text { brain }}}{A U C_{\text {tot }, \text { plasma }}}=\frac{A U C_{u, \text { brain }} \times f_{u, p l a s m a}}{A U C_{u, p l a s m a} \times f_{u, \text { brain }}},
$$

where "tot" refers to the total brain tissue or plasma drug concentrations, $f_{u, p l a s m a}$ refers to the unbound-drug fraction in plasma, and $f_{u, b r a i n}$ refers to the unbound-drug fraction in brain tissue homogenates. 


\section{Fate of drugs within the brain}

After entering across the $\mathrm{BBB}$, the drug distributes throughout the interstitial fluid space of the brain ECF into the neurons and glial cells. As more than $95 \%$ of the abluminal endothelial wall is covered with astrocyte feet, it is likely that drugs are distributed into the astrocytes early in the process. Within the brain, the drug molecules bind specifically to receptors or nonspecifically to other brain tissue components. More lipophilic compounds tend to bind nonspecifically to brain parenchymal components to a large extent. Through brain homogenate and brain slice measurements, it has been found that the ratio of total to unbound drugs varies from 1:1 up to $3,000: 1 .^{27}$ Collecting information about the relationship between total and unbound-drug concentrations in brain tissue is, therefore, crucial for obtaining an estimate of the active unbounddrug concentrations. Relying only on total concentrations can otherwise cause estimations to be up to 3,000-fold wrong.

Intracellular distribution is dependent on the $\mathrm{pKa}$ of the drug in relation to the subcellular $\mathrm{pH}$ gradients in the brain cells. Bases with high $\mathrm{pKa}$ values tend to accumulate in acidic subcellular components like lysosomes with their $\mathrm{pH}$ of 5 , in comparison with the intracellular cytosolic $\mathrm{pH}$ of 7.0, and may, therefore, act as a sink. Acids on the other hand tend to stay predominantly in the ECF.

Most compounds rapidly equilibrate across the $\mathrm{BBB}$, while a few have a slower concentration-time profile in the brain. ${ }^{28}$ The half-life of a drug in the brain will be influenced by both the extent of its binding in the brain parenchyma and the capacity of the efflux process, just as the half-life in the body is determined by the volume of distribution and the clearance (for further reading, see Hammarlund-Udenaes ${ }^{29}$ ). The unbound-drug brain concentration-time profile is also determined by the plasma concentration profile. The slowest of the elimination processes in brain or in plasma will subsequently determine the observed halflife in the brain. ${ }^{30}$

Drug transport to the CSF takes place mainly at the BCSFB, while a small part comes from the bulk flow in the brain, emptying into the CSF.

\section{ADVANCED APPROACHES FOR STUDYING BBB TRANSPORT AND INTRABRAIN DISTRIBUTION}

There is a need to direct methodologies used in discovery/ development toward measuring the unbound-drug moieties to be able to relate to pharmacological action, as elegantly shown for dopamine $\mathrm{D}_{2}$ occupancy and a few other drugs. ${ }^{31,32}$ Awareness of which parameters to focus on for furthering the success rate is increasing, and methods for use in discovery and development settings are being developed. Methods that measure the rate vs. the extent of transport have also been clarified, ${ }^{6}$ in contrast to the previous rather confused understanding evidenced in the literature. ${ }^{33}$ The methods range from simple rapid methods for the discovery/development phase to more expensive, intricate methods, such as positron emission tomography (PET) in the clinic.

\section{Microdialysis}

Microdialysis is the most sensitive method available for studying the $\mathrm{BBB}$ transport of drugs in vivo. ${ }^{34,35}$ To date, no other tech- nique has been able to obtain such quantitative and time-resolved information on the unbound drug of interest. However, while being much used in experimental settings in laboratory animals, the technique is much less used in human brain studies, apart from in patients under neurointensive care where microdialysis catheters are placed in the brain on a routine basis for clinical monitoring. ${ }^{36}$

With the sequential collection of perfusate from well-defined brain sites and blood, intricate information can be obtained on $\mathrm{BBB}$ transport. Data from microdialysis has been influential in improving our understanding of $\mathrm{BBB}$ transport and brain distribution of drugs. With this technique it has been possible to map unbound-drug concentrations over time in the brain in relation to those in blood, leading to the new concepts and measurement methods for studying $\mathrm{BBB}$ transport. ${ }^{3,6}$ However, although it is sensitive, the method does have shortcomings that limit its use. The first problem is time. The very detailed information obtained is time-consuming to gather and to analyze chemically, as many samples are collected within one study. Microdialysis is, therefore, suitable for and very valuable in academic research but it is less suitable in drug discovery/ development settings for studying drug concentrations, where more rapid information is needed. It is also much used in drug discovery for measuring drug effect in the form of transmitter release. The second problem involves the tendency for especially the more lipophilic compounds to stick to the tubings, hindering the use of the technique for drug concentration measurements for most CNS compounds.

\section{Progress in discovery/Development methods}

The Combinatory Mapping Approach uses a combination of methods to rapidly obtain important information about $\mathrm{BBB}$ transport and brain distribution of new compounds in the discovery/development setting, in a more high-throughput mode (Figure 3). ${ }^{23}$ As well as providing BBB transport information, this approach also provides information about intra- and subcellular distribution, using a limited set of experiments. By combining an in vivo measurement of $\mathrm{K}_{\mathrm{p}}$ with in vitro brain homogenate and plasma equilibrium dialysis (ED), brain slice measurements and $\mathrm{pKa}$ measurements, the $\mathrm{BBB}$ net flux $\left(\mathrm{K}_{\mathrm{p} \text {,uu,brain }}\right)$, cellular barrier transport $\left(\mathrm{K}_{\mathrm{p}, \mathrm{uu}, \mathrm{cell}}\right)$, and intra- and subcellular distribution can all be estimated (Figure 3).

In vivo measurement of the AUC in brain and plasma is normally achieved with one or a few data points. Equilibrium between plasma and brain may or may not be present at this time point, depending on the rate of $\mathrm{BBB}$ transport and brain binding properties. ${ }^{37}$ Cassette dosing, with simultaneous administration of a few compounds, has been proposed for increasing speed and saving animals, and has been found to be valuable with very little interference between compounds. ${ }^{38}$

The in vitro methods used in the Combinatory Mapping Approach are the brain homogenate ${ }^{39,40}$ and the brain slice ${ }^{41}$ methods. Brain homogenate data are generally presented as

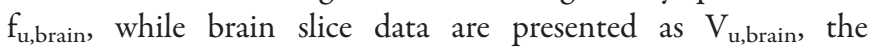
unbound-drug volume of distribution in brain, representing the relationship between the total amount and unbound-drug 


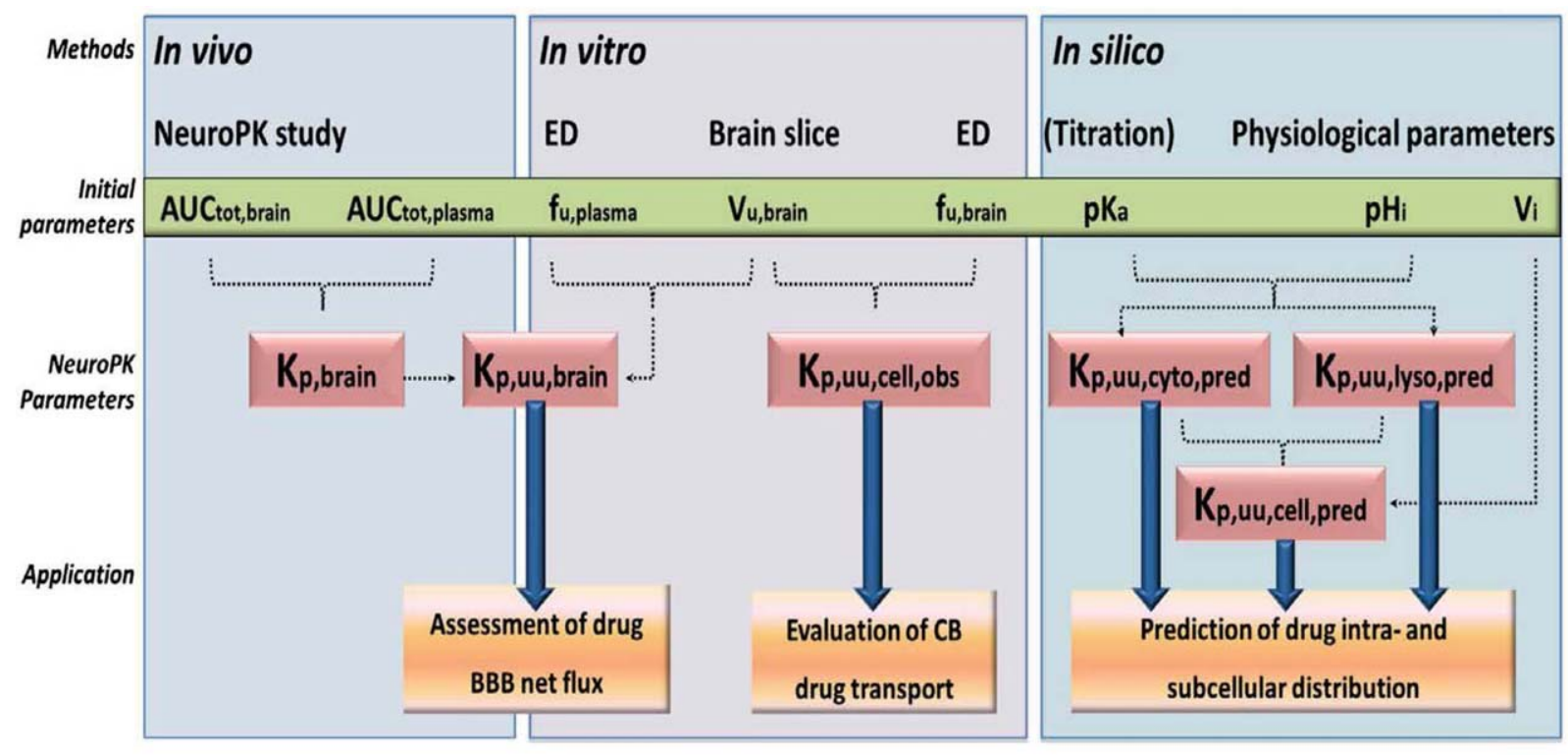

Figure 3 Combinatory mapping approach for the evaluation of unbound-drug CNS exposure in drug discovery/development. The information can be used in the selection of novel neurotherapeutics, or when avoidance of CNS side effects is wanted, when peripheral targets are present. The toolbox contains in vivo, in vitro, and in silico measurements that are combined into the calculation of parameters for evaluating the neuropharmacokinetic properties of

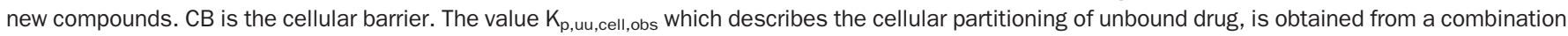
of brain slice and brain homogenate measurements (via the parameters $\mathrm{V}_{\mathrm{u} \text {,brain }}$ and $\mathrm{f}_{\mathrm{u} \text {,brain, }}$, respectively). In silico measures of pKa and estimation of physiological subcellular $\mathrm{pH}$ values $\left(\mathrm{pH}_{\mathrm{i}}\right)$ are needed for estimation of the partitioning of unbound drug between the cytosol and the extracellular fluid, pre-

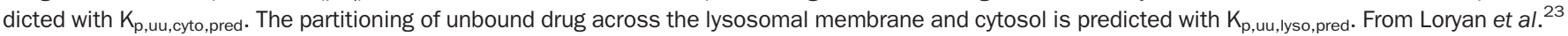
with permission from Springer.

concentration of drug in the brain parenchymal tissue. $\mathrm{V}_{\mathrm{u} \text {,brain }}$ is similar to but not equal to $1 / \mathrm{f}_{\mathrm{u} \text {,brain }}{ }^{42}$ One of the two methods is sufficient for calculating $\mathrm{K}_{\mathrm{p} \text {,uu,brain. }}$. More stable values have been obtained with the brain slice method, especially for highly bound compounds. ${ }^{42}$ Both methods can be combined for information about intracellular partitioning. The reason for this is that the brain homogenate method, with no intact cell membranes, measures overall, mainly nonspecific binding, while the brain slice method with its retained cellular barriers and subcellular $\mathrm{pH}$ differences, also measures intracellular distribution.

There are no good alternative direct measurement methods for brain drug delivery properties to date. It was recently shown that there is a clear lack of correlation between $K_{p \text {,uu,brain }}$ and $f_{u \text {,plasma }}$ for 40 CNS compounds. ${ }^{23}$ There was no correlation between partitioning across the $\mathrm{BBB}$ vs. partitioning into cells $\left(\mathrm{K}_{\mathrm{p} \text {,uu,brain }}\right.$ vs. $\left.K_{p, u u, c e l l}\right)$, nor between $K_{p, u u, b r a i n}$ and brain binding described with $\mathrm{V}_{\mathrm{u} \text {,brain }}$ from brain slice measurements. The data in the publication show that the brain and plasma binding correlation $\left(\mathrm{r}^{2}=0.66\right)$ was too small for one value to accurately predict the other. ${ }^{23}$ The explanation for why there is a lack of correlation between these entities is that the different parameters describe different types of interaction between physiology and drug physicochemical properties. Active efflux and influx transporter affin-

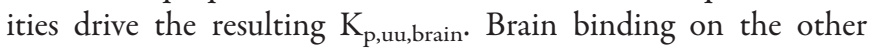
hand seems to be mainly determined by lipophilicity properties. Intracellular distribution into lysosomes is driven by $\mathrm{pKa}$ in relation to the $\mathrm{pH}$ differences between cytosol and lysosome, the latter attracting bases with high $\mathrm{pKa}$ values more than those with lower pKa values or acids. Therefore, the different properties cannot be combined into one parameter.

\section{Physicochemical properties as predictors of BBB transport and brain distribution}

Many in silico models have been developed in an effort to predict brain drug delivery. Most of them have been based on $\mathrm{K}_{\mathrm{p} \text {,brain }}$ $(\log B \mathrm{~B})$ relationships and have found lipophilicity to be the main driver for brain delivery. As discussed earlier, this has confounded the search for good predictors. Fridén et al. presented physicochemical properties that govern good $\mathrm{BBB}$ transport itself, found that the main property is the number of hydrogen bond acceptors. ${ }^{43,44}$ Very recent work on a specific CNS data set has shown that brain binding is mainly governed by the lipophilicity of the drug, while transport across the $\mathrm{BBB}$ is determined by other factors, confirming the role of hydrogen bond donors and acceptors. ${ }^{23,45}$ Highly lipophilic compounds have a tendency to be substrates for efflux transport, thereby decreasing the entry into the brain of the active moiety. In conclusion, drugs aimed for the CNS should not be too lipophilic, in total contrast to earlier guidelines. When the drug industry fully adopts the new findings we may see a much increased success rate regarding brain penetration in choosing suitable compounds for the treatment of CNS diseases. ${ }^{46}$

\section{Progress in clinical methods of measuring brain drug delivery} CSF sampling. Because, for obvious ethical reasons, microdialysis cannot be used in humans for the purpose of drug development, human CSF concentrations are still considered the best possible 
approximation of unbound-drug concentrations in brain ECF. The most reliable method is to measure drug concentrations in the lumbar CSF, collected by a single lumbar puncture during continuous intravenous drug infusion. This gives stable information on the extent of equilibration across the BCSFB, but omits any information on time dependency (rate). The CSF concentration-time profile will probably lag behind the brain ECF concentrations, making single sampling early after drug administration less reliable. ${ }^{17,47}$

Sequential CSF sampling is also possible in humans, ${ }^{48}$ although the repeated withdrawal of CSF may negatively influence the physiology. This is a concern and must be taken into account. ${ }^{49}$ In rats, it is possible to perform serial CSF sampling through a permanent cannula in the cisterna magna. Thus, the time course of concentrations in CSF can be obtained in parallel with the CNS drug effects. ${ }^{50}$ Alternatively, in rats, for detailed information on CSF drug distribution, a microdialysis probe can be placed in a ventricle or in the cisterna magna for serial sampling. ${ }^{51}$ This allows the concentrations in the CSF to be followed without disturbing the CSF fluid flow.

The question of how closely CSF concentrations reflect brain ECF concentrations in different locations in the brain, in diseases, and for different drugs remains. ${ }^{43,52}$ Depending on transporter specificities, individual drugs may differ quite substantially in concentrations between the CSF and the brain ECF. ${ }^{51}$ It can, however, be concluded that CSF sampling is the best available method for obtaining close estimates of unbound-brain ECF concentrations in humans. ${ }^{53}$

Imaging techniques. Noninvasive imaging techniques produce objective data on drug effects in the living awake brain and therewith provide the ideal tool for translation from preclinical to clinical information. Moreover, with imaging methods, important time-resolution data can be obtained. Using PET, BBB transport and brain distribution of drugs can be assessed in many species. ${ }^{11,54}$ However, the information needs to be translated to unbound-drug brain concentrations, as PET measures total brain concentrations. Some information exists on species-independent nonspecific binding of drugs in the brain, which may allow animal information to be used as a translational step. ${ }^{55}$

\section{PHARMACOMETRIC MODELING OF BBB TRANSPORT, INTRABRAIN DISTRIBUTION, AND CNS DRUG EFFECTS}

With so many factors involved in the pharmacokineticpharmacodynamic relationships of CNS drugs, and the condition-dependent rate and extent of their transport, the relationships between drug dosing and CNS effects can be highly diverse in different biological systems. Thus, a relationship obtained in a particular condition will often not directly predict that in another condition. However, living mammals in essence share the same biological processes, and, therefore, knowledge of the condition-dependent rate and extent of an individual process provides a foundation for interspecies extrapolation. ${ }^{56}$

\section{Distinction between biological system and drug properties}

The pharmacokinetics of a drug in a biological system depend on both drug properties and biological system characteristics. ${ }^{19}$ Drug properties are given, but biological system properties are condition-dependent. Therefore, it is important to separate the two, and to learn how drug and biological systems together will govern the pharmacokinetics of a drug in all body compartments. This also holds for pharmacodynamics, but that is beyond the scope of this part of the paper.

With regard to pharmacokinetics, biological system properties can be parameterized in terms of physiological tissue compartments (such as, for the CNS, blood, plasma, ECF, CSF, BBB cells, BCSFB cells, brain parenchymal cells, and tissue components such as membrane lipids and lysosomes), and their physiological/ biochemical characteristics (such as blood flow, ECF bulk flow, CSF production, CSF elimination, compartmental $\mathrm{pH}$ values, transporter and metabolic enzyme expression, and functionality at brain barriers and brain cells). ${ }^{9,57}$

\section{Pharmacometric approaches}

To improve understanding of sources of intra- and interindividual differences in the pharmacokinetics and pharmacodynamics associated with CNS drugs, we need quantitative research approaches to the factors that play a role in the relationships between drug dosing, pharmacokinetics, disease conditions, and ultimate effects, at both the population and individual levels. The quality of the pharmacometric models primarily lies in the quality of the data to be modeled. This can be improved by inclusion of multiple quantitative and objective measures such as a composite biomarker for disease condition and treatment effects. $^{58}$

The PBPK mathematical modeling approach uses biological system characteristics to describe mass transport between body compartments. While total tissue concentrations have typically been used for describing the extent of drug distribution $\left(\mathrm{K}_{\mathrm{p}}\right)$ in PBPK modeling, ${ }^{4,20}$ improvements are now being made by the use of unbound-drug concentrations, which are more accurate in describing mass transport as drug binding is excluded from the transport equation, as discussed above. ${ }^{29}$ The importance of including time-resolution data should also be emphasized, ${ }^{30,47}$ altogether indicating a need for quantitative and integral research approaches. ${ }^{4,23}$

Drug properties can be determined in vitro, and will remain as the drug's properties independent of the biological system, with its particular condition in which the drug is placed. However, obviously system-dependent properties relate to the condition, which may vary between species and between subjects at different ages and/or during different disease states. ${ }^{59}$ Thus, such information is essential for extrapolation purposes.

\section{Preclinically based translational models}

There are several studies on brain distribution that do not really include physiological parameters but that nevertheless provide considerable insight into multiple brain compartmental distribution processes (reviewed by De Lange ${ }^{60}$ ). Only a few studies have used the physiological modeling approach. Kielbasa et al. explored the potential utility of PBPK modeling using rat brain microdialysis data to predict the human brain ECF pharmacokinetics of atomoxetine and duloxetine. ${ }^{61,62}$ Plasma and brain microdialysates 
a

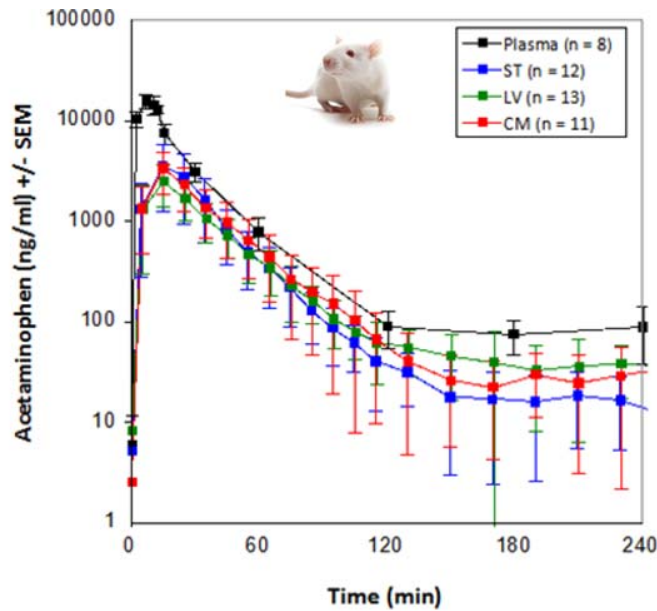

b

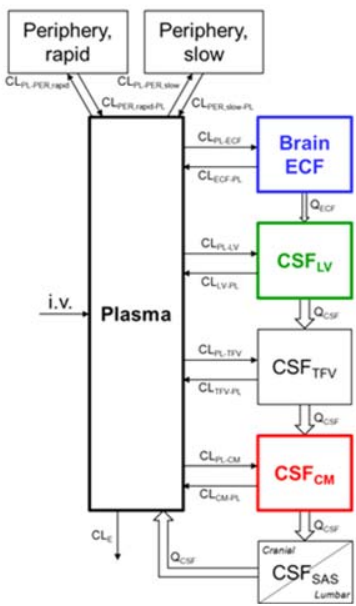

d

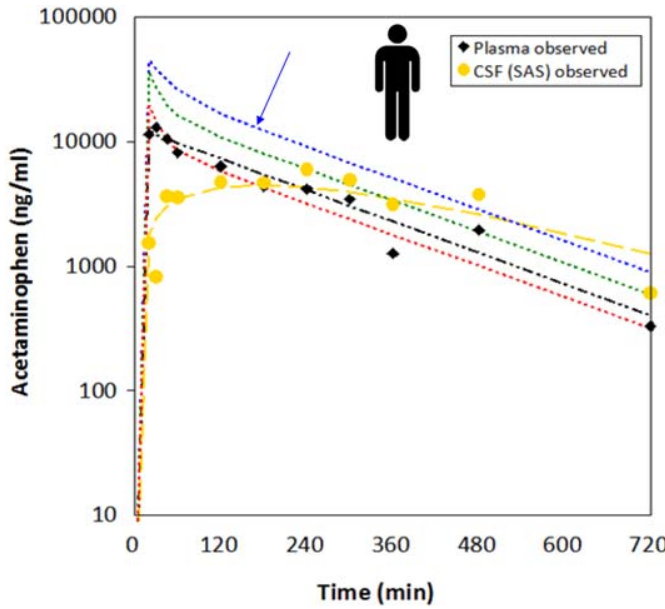

C

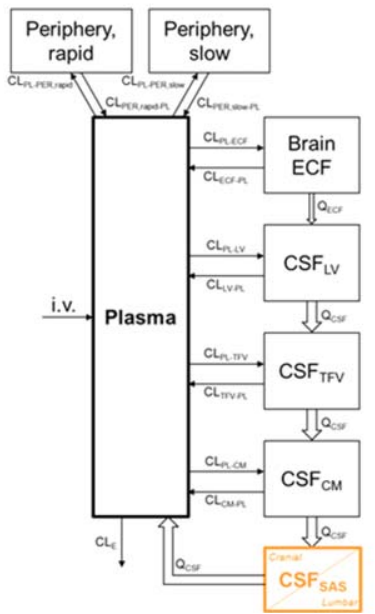

Figure 4 Translational approach for prediction of human brain drug distribution on the basis of preclinically derived data. ${ }^{63}$ (a) Concentrations of acetaminophen in plasma, brain ECF, and two CSF locations in rats. (b) Physiologically based PK model for brain distribution in rat. (c) The model tuned to human physiology. (d) The tuned model prediction of acetaminophen concentrations in plasma, brain ECF, and different CSF locations and actual human data obtained for acetaminophen in plasma and lumbar CSF. Plasma and lumbar CSF concentrations of acetaminophen were well predicted, giving confidence in the brain ECF prediction (indicated with the blue arrow). This physiological model is not drug dependent as it also holds for quinidine ${ }^{51}$ and methotrexate. ${ }^{17}$ Published with permission from Springer.

were obtained from the rat as a function of time, and were used in combination with end-of-experiment concentrations in total brain and CSF. These data were included in a model based on four brain compartments (plasma [not PBPK], brain ECF, brain cells, and CSF] and the clearances between these compartments. Nonlinear mixed-effects modeling was performed with NONMEM, was used to predict human brain ECF, brain cells, and CSF pharmacokinetics.

Westerhout et al. worked on a Mastermind Approach in their PBPK brain models; the preclinical data were produced in-house, with unbound-drug concentrations in plasma, brain ECF, brain cells, CSF in the lateral ventricle, and CSF in the cisterna magna obtained as far as possible, in parallel, in individual animals, following a short infusion of model drugs with distinct physicochemical properties. ${ }^{17,51,63}$ The model drugs acetaminophen, quinidine, and methotrexate were administered intravenously to the rat with or without concomitant administration of blockers of active transport at the brain barriers. The data were used to define the time-dependent parameters for passive and active mass exchange between plasma, brain ECF, and CSF concentrations. Physiological parameters for volumes, surfaces, fluid flows, and active transport processes in rats and humans were obtained from the literature. Modeling was all performed with NONMEM, therewith also including sources of intra- and interanimal variability on the basis of statistics.

For acetaminophen, a neutral and moderately lipophilic drug without active transport, the model successfully predicted the literature data that were obtained from healthy human plasma and lumbar CSF (Figure 4). ${ }^{63}$ For quinidine, a moderately lipophilic basic compound which is positively charged at 
physiological $\mathrm{pH}$, the influence of $\mathrm{P}$-gp-mediated transport at the brain barriers was taken into account using the P-gp blocker tariquidar. ${ }^{51}$ This study demonstrated that transporter functionality is an important factor in the relationship between CSF and brain ECF pharmacokinetics, given that the relative order of distribution of quinidine over the brain compartments changed when P-gp-mediated transport was blocked. Thus, substantial differences in the relationship between brain ECF and CSF drug distribution may result for different levels of Pgp functionality, which might, therefore, underlie mispredictions of human brain ECF concentrations on the basis of CSF measurements.

The brain distribution of methotrexate, a hydrophilic acid that undergoes Mrp/Oat/Oatp-mediated active transport, was assessed with and without inhibition by probenecid. ${ }^{17}$ The model developed for methotrexate was subsequently applied using all available literature data on brain distribution in other healthy and diseased rats (brain ECF), healthy dogs (CSF), diseased children (CSF), and diseased adults (brain ECF and CSF).

Important differences between brain ECF and CSF kinetics were found in the rat. Interestingly, inhibition of Mrp/Oat/ Oatp-mediated active transport processes, unlike P-gp inhibition as obtained for quinidine, did not significantly influence the ratio of brain ECF to CSF methotrexate concentrations. It can, therefore, be concluded that transporter function is an important factor in the relationship between CSF and brain ECF exposure, with the influence depending on the particular transporter(s) involved. This has also been observed in a more recent study for cefadroxil. ${ }^{64}$ It was possible to describe circadian variability in quinidine brain distribution in the rat with the same structural model, where higher activity of P-gp-mediated transport from the deep brain compartment to the plasma compartment was found during the active period (Figure 4). Furthermore, CSF flux (turnover) was found to be higher in the resting period. This showed that exposure of a P-gp substrate to the brain depends on the time of administration in relation to the wake-sleep cycle. ${ }^{16}$

These brain distribution studies are only the start of a new approach to translating information from rat to man; more investigations using other strategically chosen drugs with distinctively different physicochemical properties are ongoing to further develop a generic brain distribution model that is anticipated to ultimately only need input of the in vitro assessed properties of new drugs to predict their brain distribution kinetics.

\section{LINK BETWEEN BBB TRANSPORT, CNS DISEASES, AND CNS DRUG EFFECTS}

Distribution of drugs within the brain will never be completely homogeneous, so differences in concentration may exist between different brain compartments (such as brain ECF, brain [sub]cellular structures, CSF). The question remains: to which one of these concentrations is the time course of CNS drug effects most closely related? To that end, the link between drug concentrations and effect measures should be investigated. ${ }^{65}$

It is the target site pharmacokinetics that, via target binding and signal transduction is linked to the CNS drug effects. Insight into variability caused by heterogeneity (condition dependency of the biological system on condition) in target expression, target binding kinetics, target turnover, target interaction, endogenous ligand kinetics, and signal transduction processes need integral research approaches. Investigations into the pharmacokineticpharmacodynamic relationship of a CNS drug should, therefore, be designed so that the contribution of a particular process is identified, such as by systematically influencing different processes, using time-resolution methods and including unbound-drug concentration measurements, i.e., by the Mastermind Approach. ${ }^{4}$

\section{CNS diseases and targets}

The ultimate goal of using CNS drugs is to treat a disease with a target in the CNS. One of the first questions is "What can be considered as the target?" Drugs for diseases like epilepsy, Parkinson's disease, Alzheimer's disease, schizophrenia, depression, and anxiety ${ }^{13,66}$ have mostly been developed for neurotransmitter related targets, multiple sclerosis is mostly associated with immune system- and inflammation-related targets, and brain tumors, like all cancers, are mostly associated with biochemical targets that interfere with growth. However, many CNS diseases have multiple distorted components and associated targets, so speaking about one target for a disease is too simple. The focus should be on unraveling the disease fingerprint and describing the multiple targets, and if they are not measurable directly, finding biomarkers of the disease and drug effects. ${ }^{4,66}$

\section{Translational biomarkers of CNS drug effects and disease}

Human CNS drug effects are often complex and in many cases cannot be measured directly, or can be assessed only as subjective measures, i.e., with score sheets. This means that, objectively, we have to rely on biomarkers on the causal chain between drug concentration and effect. ${ }^{67}$

Moreover, given the multiple processes involved in both the drug action and the disease, it can be seen that a single biomarker will never provide enough insight: there is, therefore, a need for a combination of biomarkers (a composite or fingerprint biomarker), to be obtained at different levels of the biological system. In preclinical species, invasive experimental techniques are to a certain extent ethically allowed, but given the fact that the human brain is not really accessible for invasive measurements, these results are less translatable.

Thus, in vivo information should come from accessible body compartments like the blood and CSF sampling, and if samples are obtained from the brain, CNS noninvasive techniques should be used. These techniques can all be used in animals as well as in humans and are very valuable as they may be included in translational approaches to aid prediction (Table I). ${ }^{4,68}$

CSF. CSF fluid is the only accessible fluid in the human CNS, and for that reason it is very valuable, not only for pharmacokinetic insights into the distribution of drugs into the brain, as discussed above, but also for assessing biomarkers of disease and thereby treatment effects. ${ }^{69,70}$

Blood/Plasma hormone levels. Another, less expensive and more useful approach used both preclinically and in humans, is blood 
sampling, for measuring both drug concentrations and pharmacodynamic markers. The most important function of the hypothalamus is to link the nervous system to the endocrine system via the pituitary gland. As the brain is in constant endocrinal communication with the rest of the body, plasma samples can provide very useful information on brain functioning. ${ }^{71}$

Postmortem/Proteomics. Quantitative Targeted Absolute Proteomics (QTAP) has enabled the determination of absolute expression levels (moles) of any target protein, including low-abundance functional proteins, such as transporters and receptors. It is a promising technology for investigation of proteins, posttranslational modifications, polymorphisms, and molecular interactions in a variety of biological systems and it is, therefore, important that it is included in integrative studies. This will further our understanding of the roles of individual proteins and their differences, such as in normal or diseased conditions, in postmortem animal and human brain tissue, and in in vitro biological systems. ${ }^{72}$

Noninvasive techniques. Noninvasive imaging techniques include PET, magnetic resonance (MR)-based techniques, and electroencephalographic (EEG) techniques. These techniques produce objective data on drug effects in the living awake brain and may provide the ideal tool for translation from preclinical to clinical investigations. Moreover, important time-resolution data can be obtained with imaging methods. ${ }^{73}$ Using PET in addition to pharmacokinetic parameters on brain distribution, receptor occupancy of specific drug receptors can be assessed in normal and diseased conditions. ${ }^{5,74}$

The function of neurotransmitter pathways can be investigated with MR-based tools. Over the past three decades, several MRbased tools have yielded findings that provide tangible evidence of the neurobiological manifestations of psychiatric diseases. This promises to reveal more of the neurobiological basis of CNS disorders and also to enhance our understanding of healthy human behavior. ${ }^{75,76}$ New techniques in functional MR imaging (fMRI) promise to be part of a sequence that could transform drug development for disorders of the CNS by examining brain systems and their functional activation dynamically. ${ }^{77,78}$

Another interesting but controversial technique is the quantitative EEG (QEEG) (also termed pharmaco-EEG when drug treatment is evaluated), in which changes in parameters such as delta and theta activity, mean frequency, and coherence are determined. QEEG is able to provide direct data relating to neural activity that may be abnormal in certain disorders or changed by drug treatment. There is growing evidence that the EEG in certain circumstances can be used to predict which patients are likely to respond to treatment, thus potentially increasing the power of studies by decreasing nonresponse rates and increasing mean changes in outcome measure.

Prichep suggests that the clinical utility of EEG, especially in psychiatric or learning and cognitive disorders, has been greatly enhanced by the use of QEEG, but also emphasizes that inclusion/exclusion criteria, and adequate sampling of artifact-free data to demonstrate reliability and reproducibility of norms, spec- ificity and sensitivity should be carefully considered. ${ }^{79}$ An interesting application of QEEG is in the assessment of chronic pain. Neuroimaging literature on chronic pain demonstrates increased cerebral blood flow and metabolism consistent with increased neuronal activity in the structures comprising the "pain matrix"; furthermore, some of these regions have been shown to encode pain intensity. ${ }^{80}$ Alhaj et al. indicated that QEEG is able to provide direct data relating to neural activity that may be abnormal in certain disorders. ${ }^{81}$

\section{Translational modeling approaches}

The PBPK modeling approach has provided the basis for interspecies extrapolation, has focused on quantitative modeling of mass transport into and out of physiological compartments, and has made highly significant contributions to our knowledge of systems and the fates of drugs. This approach can be taken a step further by connecting pharmacodynamic data and data on the course of the disease to the model. The currently available models do not all include full PBPK combined with pharmacodynamic data, but they have already provided important insights into the link to human CNS drug effects, and paved the way for future more mechanistic studies that will further improve the efficacy of CNS drugs in humans. ${ }^{82,83} \mathrm{~A}$ study by Stevens et al. provides an example of the use of a plasma biomarker of a CNS drug effect; a translational model was developed on the basis of preclinical and literature data only, importantly including information on the time course of unbound-drug (the dopaminergic drug remoxipride) concentrations in the rat brain and of plasma prolactin concentrations. The model was able to predict the human plasma pharmacokinetics of remoxipride and plasma prolactin concentrations as CNS effects. ${ }^{68}$

\section{PERSPECTIVES AND IMPLICATIONS FOR FUTURE DRUG DEVELOPMENT}

The findings discussed in this review show that we have come quite a way in understanding the pharmacokinetics of BBB transport and brain distribution of drugs. However, we still lack translational information between rodents and humans, although some progress has been made here as well, particularly in the measurement of transporter proteins in the $\mathrm{BBB}$. The influence of CNS diseases on BBB transport and the intrabrain and target site distribution of drugs, including how far we can get with better use and further development of animal models of CNS diseases, also requires more work. With approaches like the Combinatory Mapping Approach in early discovery/ development and the Mastermind Approach for understanding drug behavior and influences in the biological system, together with new methodologies for measuring drug effects, there is a possibility of a higher success rate. With the distinction between drug and biological system properties now emphasized, appropriate data and advanced pharmacometrics are needed to quantitatively characterize, understand, and predict the drug's pharmacokinetic, pharmacodynamic, and biomarker outcomes, with special emphasis on sources of variability. This will aid efficient drug development, help regulatory decisions, and encourage rational, more individualized drug treatment. What remains is the full adoption of these new concepts by the drug industry, 
Table 1 Description, key aspects and (examples of) applications for CSF sampling and non-invasive techniques that may provide quantitative and time-course data on CNS PK and (biomarkers of) PD and/or disease

\begin{tabular}{|c|c|c|}
\hline Technique & Description \& key aspects & Applications \\
\hline \multicolumn{3}{|l|}{$\begin{array}{l}\text { Fluid } \\
\text { based }\end{array}$} \\
\hline $\begin{array}{l}\text { CSF } \\
\text { sampling }\end{array}$ & $\begin{array}{l}\text { Cerebrospinal fluid (CSF) samples can be obtained from ven- } \\
\text { tricles or lumbar region of the spine. CSF is in contact with brain } \\
\text { parenchyma and may therefore to a certain level reflect proc- } \\
\text { esses of the brain parenchyma. }\end{array}$ & $\begin{array}{l}\text { T-tau levels (total tau, tau and phosphorylated tau) as a marker of } \\
\text { neuronal damage for example for (not-exclusive) diagnosis of } \\
\text { Alzheimers disease } \\
\text { Amyloid beta42 levels as marker for level of plaque sequestra- } \\
\text { tion, for example for (not-exclusive) diagnosis of Alzheimers } \\
\text { disease } \\
\text { Alpha-synuclein for pathogenesis of synucleinopathies, for exam- } \\
\text { ple for (not-exclusive) diagnosis of Parkinson's disease } \\
\text { Urate, a potent antioxidant, seems to be associated to the risk of } \\
\text { developing Parkinson's disease }\end{array}$ \\
\hline
\end{tabular}

MRI Magnetic resonance imaging (MRI) uses the body's natural magnetic properties to produce detailed image. The hydrogen nucleus (proton, ${ }^{1} \mathrm{H}$ ) is mostly used because of its abundance in water and fat, that behaves like a small bar magnet. Under normal circumstances, these hydrogen proton "bar magnets" spin in the body with their axes randomly aligned. When the body is placed in a strong magnetic field, such as an MRI scanner, the protons' axes all line up. This uniform alignment creates a magnetic vector oriented along the axis of the MRI scanner. When additional energy (in the form of a radio wave) is added to the magnetic field, the magnetic vector is deflected. The radio wave frequency (RF) that causes the hydrogen nuclei to resonate is dependent on the element sought (hydrogen in this case) and the strength of the (gradiented) magnetic field. When the radiofrequency source is switched off the magnetic vector returns to its resting state, and this causes a signal (also a radio wave) to be emitted. It is this signal which is used to create the MR images.

fMRI Functional magnetic resonance imaging, or fMRI, is a technique for measuring brain activity. It works by detecting the changes in blood oxygenation and flow that occur in response to neural activity - when a brain area is more active it consumes more oxygen and to meet this increased demand blood flow increases to the active area.

Resting state networks (RSNs)
$\mathrm{N}$-acetylaspartate (NAA) appears in a prominent peak in ${ }^{1} \mathrm{H}$-MRS. Exhibition by NAA of time-dependent attenuation that reflects energy metabolism during the acute stage of cerebral ischemia makes this metabolite a unique biomarker for assessing ischemic stroke.

Other major metabolites detected with ${ }^{1} \mathrm{H}-\mathrm{MRS}$ include, total cholines (tCho), total creatines (tCr), lactate (Lac), mobile lipids (Lip) and other macromolecules (MM), myo-inositol (ml) and glutamine and glutamate (Glx). The tCr signal arises from the adenosine diphosphate-adenosine triphosphate energy cycle metabolites creatine and phosphocreatine and is a marker for cellular energy metabolism.

$\mathrm{MRI}$ is the investigative tool of choice for neurological cancers as it is more sensitive than CT for small tumors and offers better visualization of the posterior fossa. The contrast provided between grey and white matter makes it the optimal choice for many conditions of the central nervous system including demyelinating diseases, dementia, cerebrovascular disease, infectious diseases and epilepsy.

The blood oxygenation level dependence (BOLD) is the main approach to measure brain activity through fMRI. Based on an interplay of local brain blood flow and Volume associated with neuronal activity assesses indirectly the level of neuronal responses to stimuli, such as sensory or pharmacological inputs. Effectiveness of pharmacological treatments in patients can be evaluated across CNS disease-related function or differentiation of drugs or drug dosing.

One of the exciting development is the use of BOLD to measure RSNs; these are low-level spontaneous fluctuations in the BOLD signal during rest that reflect the neuronal baseline activity of the brain. As such, they can differentiate disease state or drug effects. Resting state fMRI studies in epilepsy have derived information with regards to network dysfunction within and across epilepsy syndromes

Connectivity measurements

\begin{abstract}
Repeated BOLD measurements of brain activity have been utilized to determine brain networks associated with basal brain function as well as alteration in the disease state or drug effects Recently, series of basal networks in the healthy state have been identified and their modification in different diseases established. Inspection of the functional role of each of these networks permits correlate observed behavioral outcomes in diseases and the concomitant correction of these changes when disease is resolved or ameliorated.
\end{abstract}


Table 1 Continued

\begin{tabular}{lll}
\hline Technique & Description \& key aspects & Applications \\
\hline Pharmacological fMRI (phMRI) & phMRI is variant of fMRI and refers to a particular approach of \\
& fMRI in which the specific brain response to a CNS-acting drug is & measured.
\end{tabular}

Arterial spin labeling (ASL)

Arterial spin labeling is a technique that allows the measure of perfusion without the use of external agents (such as contrast agents). ${ }^{30}$ More quantitative than BOLD but with less spatial and temporal resolution permits to capture snapshots of basal perfusion and if necessary brain activity. Quantitative pharmacologica effects on brain activation can be evaluated using this approach.

Morphometric measurements

Voxel base measures (VBM) and surface base methods allow the examination of changes in brain structures in volume and thickness. Such measures have shown that, in the disease state, dilation and shrinkage occur and that treatment can resolve these changes. Disease state ${ }^{31}$ and long-term effects of drugs on brain morphometry may be evaluated using this approach. ${ }^{32}$

Diffusion tensor imaging (DTI)

DTI is an MRI technique that measures the apparent diffusion coefficient of water molecules in tissue. Improvements in the ability to capture DTI data and analysis tools have resulted in new measurements of white matter integrity as well as determine potential tracks among different brain regions. Drugs (for example, ketamine or opioids) may produce changes in white matter tracts $^{33}$ that may reflect either direct actions or effects on neuronal bodies

Magnetic resonance spectroscopy (MRS) provides measures of
brain neurochemistry (metabolites such as amino acids, neuroChemical measures can provide insights into drug modulation of excitatory and inhibitory neurotransmitters systems and the transmitters, and so on). potential to predict responders and non-responders

Phosphor magnetic resonance spectroscopy ( $\left.{ }^{31} \mathrm{P}-\mathrm{MRS}\right)$ can inform on intracellular energy stores. The signals from the three phosphorus atoms in adenosine triphosphate and phosphocreatine vary with intracellular $\mathrm{pH}$, and from phosphomonoesters and phosphodiesters.

VBM Magnetic Morphometric measurements (Voxel base measures, VBM) and surface base methods allow the examination of changes in brain structures in volume and thickness.

\begin{tabular}{ll}
\hline $\begin{array}{l}\text { X-ray } \\
\text { based }\end{array}$ & Description \\
\hline $\mathbf{C T}$ & $\begin{array}{l}\text { Computed tomography (CT) imaging is based on the differential } \\
\text { absorption of X-rays. Bone and hard tissue absorb x-rays well, air } \\
\text { and water absorb very little and soft tissue is somewhere in } \\
\text { between. }\end{array}$
\end{tabular}

CT may be done with or without IV contrast.
MRS has been used for many years to study the metabolism of tumor cells. These signals can show changes in malignant tumors.
In the disease state, dilation and shrinkage may occur and treatment can resolve these changes. Disease state and long-term effects of drugs on brain morphometry may be evaluated using this approach. For example the shrinkage of especially the hippocampus in Alzheimer's disease.
Application

CT scans can clearly show very small bones and surrounding tissues such as muscle and blood vessels

Noncontrast CT is used to detect acute hemorrhage in the brain, as well as to characterize tissue structures.

IV contrast is used to improve imaging of tumors, infection, inflammation, and trauma in soft tissues and to assess the vascular system

\begin{tabular}{|c|c|c|}
\hline $\begin{array}{l}\text { Positrom } \\
\text { emission } \\
\text { based }\end{array}$ & Description & Application \\
\hline PET & $\begin{array}{l}\text { Positron Emission Tomography (PET) uses trace amounts of } \\
\text { short-lived radioactive material to map functional processes in } \\
\text { the brain. When the material undergoes radioactive decay a posi- } \\
\text { tron is emitted, which can be picked up be the detector. }\end{array}$ & $\begin{array}{l}\text { Biodistribution } \\
\text { Cerebral blood flow } \\
\text { BBB function } \\
\text { Target occupancy } \\
\text { Glucose utlization } \\
\text { Pharmacodynamic responses } \\
\text { Disease conditions }\end{array}$ \\
\hline
\end{tabular}


Table 1 Continued

Technique Description \& key aspects

Target occupancy

\section{Applications}

Quantitative imaging of the $D_{2}$ receptor in the basal ganglia using $\left[{ }^{11} \mathrm{C}\right]$ raclopride showed that the magnitude of its blockade by a range of antipsychotic drugs correlated with symptom relief, thereby supporting the dopamine hypotheses of antipsychotic action

Receptor occupancy titrated against plasma concentrations of antipsychotic drugs showed distinct thresholds for antipsychotic effects and extrapyramidal syndromes

The development of novel therapeutics for mood disorders has benefited from the assessment of target occupancy with PET and has been used in the development of 5- $\mathrm{HT}_{1 \mathrm{~A}}$ antagonists, dopamine reuptake inhibitors, and MAO-A inhibitors

Facilitated by the development of suitable radioligands for monoaminergic receptors (such as the $5-\mathrm{HT}_{1 \mathrm{~A}}, 5-\mathrm{HT}_{2 \mathrm{~A}}$, and $\mathrm{D}_{2}$ ), reuptake transporters (such as the serotonin transporter (SERT/5HTT), dopamine transporter (DAT) and norepinephrine transporter $(\mathrm{NET})$ ), and the catabolic enzymes (MAO-A and -B), PET imaging has had a considerable impact on the monoamine theory of major depression.

Pharmacodynamic biomarkers for proof of pharmacology

$\left[{ }^{18} \mathrm{~F}\right]-6$-fluoro-L- 3,4-dihydroxyphenylalanine (FDOPA), $\left[{ }^{18} \mathrm{~F}\right]-3^{\prime}$-fluorothymidine (FLT)) can be used to assess specific metabolic or synthetic rates, allowing inferences concerning the functional state or integrity of a tissue

Glucose utilization

$\left[{ }^{18} \mathrm{~F}\right] \mathrm{FDG}$ is used to measure absolute regional cerebral glucose utilization rate (in $\mathrm{mg} / \mathrm{g}$ per minute).It can be applied for tumor localization throughout the body, principally for disease staging and also as a marker of treatment response.

BBB function

Using $\left[{ }^{11} \mathrm{C}\right]$ verapamil, PET imaging has shown that P-gp function is decreased in AD. This is the first direct evidence that the P-gp transporter at the BBB is compromised in sporadic AD, suggesting that it may be involved in $A D$ pathogenesis

The increased ${ }^{11} \mathrm{C}$-MET uptake (factor of 1.3-3.5 compared with a contralateral control region) is related to increased transport mediated by type L-amino acid transporters located at the bloodbrain barrier (BBB).

Cerebral blood flow (CBF)

The Steady-state oxygen-15 method is a quantitative application for the determination of the regional cerebral metabolic rate for oxygen metabolism $\left(\mathrm{CMRO}_{2}\right)$ and cerebral blood flow (CBF) Oxygen extraction fraction (OEF), has been studied in patients with recurrent cerebral ischemic attacks

Disease conditions

The first-generation TSPO ligand $\left[{ }^{11} \mathrm{C}\right] \mathrm{PK} 11195$ has been used as a marker of activated microglia to show increased neuroinflammatory activity in $A D$ patients

${ }^{11} \mathrm{C}$-TYR uptake represents both transport and protein synthesis, Ligands that target amyloid deposits, such as the $\left[{ }^{11} \mathrm{C}\right] \mathrm{PiB}$ binds selectively to fibrillary amyloid, are used to distinguished $A D$ patients from age-matched controls

$\left[{ }^{18} \mathrm{~F}\right]$ fluoromisonidazole is used to image tumor hypoxia In neuro-oncology, radiolabelled methionine and thymidine compounds have been shown to be more specific tracers in tumour detection, delineation and staging owing to their relatively low uptake in normal brain.

Differentiation of AD from DLB is readily shown by PET imaging of presynaptic nigrostraital dopamine terminal integrity with $\left[{ }^{18} \mathrm{~F}\right] \mathrm{F}$ DOPA or the striatal vesicular monoamine transporter marker $\left[{ }^{11} \mathrm{C}\right] \mathrm{DTBZ}$

$\left.{ }^{11} \mathrm{C}\right] \mathrm{FMZ}$ delineation of $\gamma$-aminobutyric acid receptor A (GABA-A) availability may provide a biochemical marker of epileptogenicity and strengthens the hypothesis that inhibitory mechanisms are disturbed in the epileptic focus

$\left[{ }^{18} \mathrm{~F}\right]$ galacto-RGD peptide has been used to image angiogenesis in brain tumors 
Table 1 Continued

\begin{tabular}{|c|c|c|}
\hline Technique & Description \& key aspects & Applications \\
\hline \multicolumn{3}{|l|}{$\begin{array}{l}\text { Electrical } \\
\text { signal } \\
\text { based }\end{array}$} \\
\hline qEEG & $\begin{array}{l}\text { Electroencephalography (EEG) is the measurement of the electri- } \\
\text { cal activity of the brain by recording from electrodes placed on } \\
\text { the scalp. The resulting traces are known as an electroencepha- } \\
\text { logram (EEG) and represent an electrical signal from a large num- } \\
\text { ber of neurons.It enables the study of the electrophysiology of } \\
\text { the brain with high temporal resolution. Several analytical } \\
\text { approaches to extract information from the EEG signal have been } \\
\text { proposed. }\end{array}$ & $\begin{array}{l}\text { The EEG is capable of detecting changes in electrical activity in } \\
\text { the brain on a millisecond-level. It is one of the few techniques } \\
\text { available that has such high temporal resolution. } \\
\text { Quantitative EEG produces images of both excitatory and inhibi- } \\
\text { tory cortical neuronal activity rather than secondary hemody- } \\
\text { namic processes, and the spectra can be displayed as statistical } \\
\text { probability maps in which brain areas can be made to "light up" } \\
\text { in proportion to the abnormality of their activity. } \\
\text { REM sleep reduction can be quantified for antidepressants } \\
\text { Spikes and waves can be quantified for antiepileptics } \\
\text { EEG beta power can be used to visualize effects of GABAergic } \\
\text { anxiolytics }\end{array}$ \\
\hline
\end{tabular}

Microstate analysis, considers the multichannel EEG recording as a series of quasi-stable "microstates" that are each characterized by a unique topography of electric potentials over the entire channel array. By nature, EEG is a potential method to assess neuropsychiatric disorders since it reflects summated electrical activity at the level of functional units of the brain with millisecond time resolution.
Because this technique simultaneously considers signals recorded from all areas of the cortex, it is capable of assessing the function of large-scale brain networks whose disruption is associated with several neuropsychiatric disorders. all the way from medicinal chemistry to the clinic. One of the biggest hurdles seems to be the time required for educational exchanges between different professions, something society appears not to have the patience for, given the immense need for new treatments for CNS diseases. The closure of CNSfocused research programs by several companies during recent years also indicates that the area needs more rapid improvement to fulfill societal needs.

\section{AUTHOR CONTRIBUTIONS}

E. C. M. d.L. and M. H. U. wrote the manuscript.

\section{CONFLICTS OF INTEREST}

E.C.M.d.L. is Associate Professor in Pharmacology at Leiden University, the Netherlands. She is an occasional consultant for several pharmaceutical companies. No patents involved. No stock ownership or options are involved. From the TIPharma PKPD modeling Platform consortium, an academic setting, there is collaboration between Leiden and Groningen University and Jansen Pharmaceutica, Astellas and Takeda, from which one PhD student (May 2013-May 2017) and one Post-doc (May 2013May 2015) are supported in the CNS area. M.H.U. is a Professor in Pharmacokinetics and Pharmacodynamics at Uppsala University, Sweden. She is consultant on an individual time basis for several drug companies, including Janssen Research, Novartis, Astra-Zeneca, Roche, Grünenthal, Orion Pharma, Biogen Idec and Boehringer-Ingelheim. No patents. No stock ownership or options are involved. One post-doc student is being supported by Astra Zeneca during 2013-2015 (2 y).

(C) 2015 American Society for Clinical Pharmacology and Therapeutics
1. Hurko, O. \& Ryan, J.L. Translational research in central nervous system drug discovery. NeuroRx 2, 671-682 (2005).

2. Hammarlund-Udenaes, M., Bredberg, U. \& Friden, M. Methodologies to assess brain drug delivery in lead optimization. Curr. Top. Med. Chem. 9, 148-162 (2009).

3. de Lange, E.C., Ravenstijn, P.G., Groenendaal, D. \& van Steeg, T.J. Toward the prediction of CNS drug-effect profiles in physiological and pathological conditions using microdialysis and mechanism-based pharmacokinetic-pharmacodynamic modeling. AAPS J. 7, E532-E543 (2005).

4. de Lange, E.C. The mastermind approach to CNS drug therapy: translational prediction of human brain distribution, target site kinetics, and therapeutic effects. Fluids Barriers CNS 10, 12 (2013).

5. Abbott, N.J., Patabendige, A.A., Dolman, D.E., Yusof, S.R. \& Begley, D.J. Structure and function of the blood-brain barrier. Neurobiol. Dis. 37, 13-25 (2010).

6. Hammarlund-Udenaes, M., Friden, M., Syvanen, S. \& Gupta, A. On the rate and extent of drug delivery to the brain. Pharm. Res. 25, 1737-1750 (2008).

7. de Lange, E.C. Potential role of $A B C$ transporters as a detoxification system at the blood-CSF barrier. Adv. Drug Deliv. Rev. 56, 17931809 (2004).

8. Lee, G., Dallas, S., Hong, M. \& Bendayan, R. Drug transporters in the central nervous system: brain barriers and brain parenchyma considerations. Pharmacol. Rev. 53, 569-596 (2001).

9. Shawahna, R. et al. Transcriptomic and quantitative proteomic analysis of transporters and drug metabolizing enzymes in freshly isolated human brain microvessels. Mol. Pharm. 8, 1332-1341 (2011).

10. Dauchy, S. et al. ABC transporters, cytochromes P450 and their main transcription factors: expression at the human blood-brain barrier. J. Neurochem. 107, 1518-1528 (2008).

11. Syvanen, S. et al. Species differences in blood-brain barrier transport of three positron emission tomography radioligands with emphasis on P-glycoprotein transport. Drug Metab. Dispos. 37, 635-643 (2009).

12. van Assema, D.M. et al. P-glycoprotein function at the blood-brain barrier: effects of age and gender. Mol. Imaging Biol. 14, 771-776 (2012).

13. De Lange, E.C. Disease influence on BBB transport in neurodegenerative disorders. In Drug Delivery to the Brain. Physiological Concepts, Methodologies, and Approaches (eds. Hammarlund-Udenaes, M. 
et al.) 591-634 (Springer, New York, Heidelberg, Dordrecht, London, 2014).

14. Mehta, D.C., Short, J.L., Hilmer, S.N. \& Nicolazzo, J.A. Drug access to the central nervous system in Alzheimer's disease: preclinical and clinical insights. Pharm. Res. (2014); e-pub ahead of print.

15. Mulder, M. et al. Apolipoprotein E protects against neuropathology induced by a high-fat diet and maintains the integrity of the bloodbrain barrier during aging. Lab. Invest. 81, 953-960 (2001).

16. Kervezee, L. et al. Diurnal variation in P-glycoprotein-mediated transport and cerebrospinal fluid turnover in the brain. AAPS J. 16, 10291037 (2014)

17. Westerhout, J., van den Berg, D.J., Hartman, R., Danhof, M. \& de Lange, E.C. Prediction of methotrexate CNS distribution in different species - influence of disease conditions. Eur. J. Pharm. Sci. 57, 1124 (2014).

18. Ravenstijn, P.G., Drenth, H.J., O’Neill, M.J., Danhof, M. \& De Lange, E.C. Evaluation of blood-brain barrier transport and CNS drug metabolism in diseased and control brain after intravenous L-DOPA in a unilateral rat model of Parkinson's disease. Fluids Barriers CNS 9, 4 (2012).

19. Dedrick, R.L. \& Bischoff, K.B. Species similarities in pharmacokinetics. Fed. Proc. 39, 54-59 (1980).

20. Rowland, M., Peck, C. \& Tucker, G. Physiologically-based pharmacokinetics in drug development and regulatory science. Annu. Rev. Pharmacol. Toxicol. 51, 45-73 (2011).

21. Declèves, X., Strazielle, N., Scherrmann, J.M. \& Ghersi-Egea, J.F. Drug metabolism at the blood-brain and blood-CSF barriers. In Drug Delviery to the Brain. Physiological Concepts, Methodologies and Approaches (eds. Hammarlund-Udenaes, M. et al.) 101-124 (Springer, New York, Heidelberg, Dordrecht, London, 2014).

22. Takasato, Y., Rapoport, S.I. \& Smith, Q.R. An in situ brain perfusion technique to study cerebrovascular transport in the rat. Am. J. Physiol. 247, H484-H493 (1984).

23. Loryan, I. et al. Mechanistic understanding of brain drug disposition to optimize the selection of potential neurotherapeutics in drug discovery. Pharm. Res. 31, 2203-2219 (2014).

24. Gupta, A., Chatelain, P., Massingham, R., Jonsson, E.N. \& Hammarlund-Udenaes, M. Brain distribution of cetirizine enantiomers: comparison of three different tissue-to-plasma partition coefficients: $\mathrm{K}(\mathrm{p}), \mathrm{K}(\mathrm{p}, \mathrm{u})$, and $\mathrm{K}(\mathrm{p}, \mathrm{uu})$. Drug Metab. Dispos. 34, 318-323 (2006).

25. Deo, A.K., Theil, F.P. \& Nicolas, J.M. Confounding parameters in preclinical assessment of blood-brain barrier permeation: an overview with emphasis on species differences and effect of disease states. Mol. Pharm. 10, 1581-1595 (2013).

26. Summerfield, S.G. et al. Improving the in vitro prediction of in vivo central nervous system penetration: integrating permeability, Pglycoprotein efflux, and free fractions in blood and brain. $J$. Pharmacol. Exp. Ther. 316, 1282-1290 (2006).

27. Friden, M. et al. Development of a high-throughput brain slice method for studying drug distribution in the central nervous system. Drug Metab. Dispos. 37, 1226-1233 (2009).

28. Liu, X. et al. Use of a physiologically based pharmacokinetic model to study the time to reach brain equilibrium: an experimental analysis of the role of blood-brain barrier permeability, plasma protein binding, and brain tissue binding. J. Pharmacol. Exp. Ther. 313, 1254-1262 (2005).

29. Hammarlund-Udenaes, M. Pharmacokinetic concepts in brain drug delivery. In Drug Delivery to the Brain. Physiological Concepts, Methodologies and Approaches (eds. Hammarlund-Udenaes, M. et al.) 127-161 (Springer, New York, Heidelberg, Dordrecht, London, 2014).

30. Hammarlund-Udenaes, M., Paalzow, L.K. \& de Lange, E.C. Drug equilibration across the blood-brain barrier-pharmacokinetic considerations based on the microdialysis method. Pharm. Res. 14, 128-134 (1997).

31. Watson, J. et al. Receptor occupancy and brain free fraction. Drug Metab. Dispos. 37, 753-760 (2009).

32. Hammarlund-Udenaes, M. Active-site concentrations of chemicals are they a better predictor of effect than plasma/organ/tissue concentrations? Basic Clin. Pharmacol. Toxicol. 106, 215-220 (2010).

33. Pardridge, W.M. $\log (\mathrm{BB})$, PS products and in silico models of drug brain penetration. [comment]. Drug Discov. Today 9, 392-393 (2004).
34. de Lange, E.C., Danhof, M., de Boer, A.G. \& Breimer, D.D. Methodological considerations of intracerebral microdialysis in pharmacokinetic studies on drug transport across the blood-brain barrier. Brain Res. Brain Res. Rev. 25, 27-49 (1997).

35. Hammarlund-Udenaes, M. The use of microdialysis in CNS drug delivery studies. Pharmacokinetic perspectives and results with analgesics and antiepileptics. Adv. Drug Deliv. Rev. 45, 283-294 (2000).

36. Ederoth, P. et al. Blood-brain barrier transport of morphine in patients with severe brain trauma. Br. J. Clin. Pharmacol. 57, 427-435 (2004).

37. Liu, X. \& Chen, C. Strategies to optimize brain penetration in drug discovery. Curr. Opin. Drug Discov. Dev. 8, 505-512 (2005).

38. Liu, X., Ding, X., Deshmukh, G., Liederer, B.M. \& Hop, C.E. Use of the cassette-dosing approach to assess brain penetration in drug discovery. Drug Metab. Dispos. 40, 963-969 (2012).

39. Kalvass, J.C. \& Maurer, T.S. Influence of nonspecific brain and plasma binding on CNS exposure: implications for rational drug discovery. Biopharm. Drug Dispos. 23, 327-338 (2002).

40. Mano, Y., Higuchi, S. \& Kamimura, H. Investigation of the high partition of YM992, a novel antidepressant, in rat brain - in vitro and in vivo evidence for the high binding in brain and the high permeability at the BBB. Biopharm. Drug Dispos. 23, 351-360 (2002).

41. Loryan, I., Friden, M. \& Hammarlund-Udenaes, M. The brain slice method for studying drug distribution in the CNS. Fluids Barriers CNS 10, 6 (2013).

42. Friden, M. et al. Measurement of unbound drug exposure in brain: modeling of $\mathrm{pH}$ partitioning explains diverging results between the brain slice and brain homogenate methods. Drug Metab. Dispos. 39, 353-362 (2011).

43. Friden, M. et al. Structure-brain exposure relationships in rat and human using a novel data set of unbound drug concentrations in brain interstitial and cerebrospinal fluids. J. Med. Chem. 52, 62336243 (2009).

44. Chen, H., Winiwarter, S., Friden, M., Antonsson, M. \& Engkvist, O. In silico prediction of unbound brain-to-plasma concentration ratio using machine learning algorithms. J. Mol. Graph. Model. 29, 985-995 (2011).

45. Loryan, I. et al. Molecular properties determining unbound intracellular and extracellular brain exposure of CNS drug candidates. Mol. Pharm. (2015); e-pub ahead of print.

46. Liu, X., Wright, M. \& Hop, C.E. Rational use of plasma protein and tissue binding data in drug design. J. Med. Chem. 57, 8238-8248 (2014).

47. Westerhout, J., Danhof, M. \& De Lange, E.C. Preclinical prediction of human brain target site concentrations: considerations in extrapolating to the clinical setting. J. Pharm. Sci. 100, 3577-3593 (2011).

48. Bruce, J., Tamarkin, L., Riedel, C., Markey, S. \& Oldfield, E. Sequential cerebrospinal fluid and plasma sampling in humans: 24hour melatonin measurements in normal subjects and after peripheral sympathectomy. J. Clin. Endocrinol. Metab. 72, 819-823 (1991).

49. Miyakawa, Y. et al. Effect of cerebrospinal fluid removal on cerebral blood flow and metabolism in the baboon: influence of tyrosine infusion and cerebral embolism on cerebrospinal fluid pressure autoregulation. Stroke 8, 346-351 (1977).

50. Danhof, M. \& Levy, G. Kinetics of drug action in disease states. I. Effect of infusion rate on phenobarbital concentrations in serum, brain and cerebrospinal fluid of normal rats at onset of loss of righting reflex. J. Pharmacol. Exp. Ther. 229, 44-50 (1984).

51. Westerhout, J., Smeets, J., Danhof, M. \& de Lange, E.C. The impact of P-gp functionality on non-steady state relationships between CSF and brain extracellular fluid. J. Pharmacokinet. Pharmacodyn. 40, 327-342 (2013).

52. de Lange, E.C. \& Danhof, M. Considerations in the use of cerebrospinal fluid pharmacokinetics to predict brain target concentrations in the clinical setting: implications of the barriers between blood and brain. Clin. Pharmacokinet. 41, 691-703 (2002).

53. de Lange, E.C. Utility of CSF in translational neuroscience. J. Pharmacokinet. Pharmacodyn. 40, 315-326 (2013).

54. Syvanen, S., Schenke, M., van den Berg, D.J., Voskuyl, R.A. \& de Lange, E.C. Alteration in P-glycoprotein functionality affects intrabrain distribution of quinidine more than brain entry-a study in rats subjected to status epilepticus by kainate. AAPS J. 14, 87-96 (2012).

55. $\mathrm{Di}$, L. et al. Species independence in brain tissue binding using brain homogenates. Drug Metab. Dispos. 39, 1270-1277 (2011). 
56. Boxenbaum, H. Interspecies scaling, allometry, physiological time, and the ground plan of pharmacokinetics. J. Pharmacokinet. Biopharm. 10, 201-227 (1982).

57. Decleves, X. et al. Interplay of drug metabolizing CYP450 enzymes and $\mathrm{ABC}$ transporters in the blood-brain barrier. Curr. Drug Metab. 12, 732-741 (2011).

58. Danhof, M., de Lange, E.C., Della Pasqua, O.E., Ploeger, B.A. \& Voskuyl, R.A. Mechanism-based pharmacokinetic-pharmacodynamic (PK-PD) modeling in translational drug research. Trends Pharmacol. Sci. 29, 186-191 (2008).

59. Collins, J.M. \& Dedrick, R.L. Distributed model for drug delivery to CSF and brain tissue. Am. J. Physiol. 245, R303-R310 (1983).

60. De Lange, E.C. Toward a generic PBPK models for brain distribution of CNS drugs. In Blood-Brain Barrier in Drug Discovery: Optimizing Brain Exposure of CNS Drugs and Minimizing Brain Side Effects (eds. Di, L. \& Kerns, E.H.) 296-323 (Wiley, New York, 2015).

61. Kielbasa, W., Kalvass, J.C. \& Stratford, R. Microdialysis evaluation of atomoxetine brain penetration and central nervous system pharmacokinetics in rats. Drug Metab. Dispos. 37, 137-142 (2009).

62. Kielbasa, W. \& Stratford, R.E. Exploratory translational modeling approach in drug development to predict human brain pharmacokinetics and pharmacologically relevant clinical doses. Drug Metab. Dispos. 40, 877-883 (2012).

63. Westerhout, J., Ploeger, B., Smeets, J., Danhof, M. \& de Lange, E.C. Physiologically based pharmacokinetic modeling to investigate regional brain distribution kinetics in rats. AAPS J. 14, 543-553 (2012).

64. Chen, X. et al. Effect of transporter inhibition on the distribution of cefadroxil in rat brain. Fluids Barriers CNS 11, 25 (2014).

65. De Lange, E.C. PKPD aspects of brain drug delivery in a translational perspective. In Drug Delivery to the Brain. Physiological Concepts, Methodologies, and Approaches (eds. Hammarlund-Udenaes, M. et al.) 233-268 (Springer, New York, Heidelberg, Dordrecht, London, 2014).

66. De Lange, E.C. Pharmacometrics in psychiatry. In Applied Pharmacometrics (eds. Schmidt, S. \& Derendorf, H.) 407-454 (Springer, New York, Heidelberg, Dordrecht, London, 2014).

67. Danhof, M., Alvan, G., Dahl, S.G., Kuhlmann, J. \& Paintaud, G. Mechanism-based pharmacokinetic-pharmacodynamic modeling-a new classification of biomarkers. Pharm. Res. 22, 1432-1437 (2005).

68. Stevens, J. et al. Mechanism-based PK-PD model for the prolactin biological system response following an acute dopamine inhibition challenge: quantitative extrapolation to humans. J. Pharmacokinet. Pharmacodyn. 39, 463-477 (2012).

69. Chou, S.H. \& Robertson, C.S. Monitoring biomarkers of cellular injury and death in acute brain injury. Neurocrit. Care (2014); e-pub ahead of print.

70. Jimenez-Jimenez, F.J., Alonso-Navarro, H., Garcia-Martin, E. \& Agundez, J.A. Cerebrospinal fluid biochemical studies in patients with
Parkinson's disease: toward a potential search for biomarkers for this disease. Front. Cell Neurosci. 8, 369 (2014).

71. Johnstone, E.C. \& Ferrier, I.N. Neuroendocrine markers of CNS drug effects. Br. J. Clin. Pharmacol. 10, 5-21 (1980).

72. Uchida, Y. et al. A study protocol for quantitative targeted absolute proteomics (QTAP) by LC-MS/MS: application for inter-strain differences in protein expression levels of transporters, receptors, claudin-5, and marker proteins at the blood-brain barrier in ddY, FVB, and C57BL/6J mice. Fluids Barriers CNS 10, 21 (2013).

73. Wintermark, M., Sanelli, P.C., Anzai, Y., Tsiouris, A.J. \& Whitlow, C.T. Imaging evidence and recommendations for traumatic brain injury: advanced neuro- and neurovascular imaging techniques. AJNR Am. J. Neuroradiol. (2014); e-pub ahead of print.

74. Johnson, M. et al. Dopamine d2 receptor occupancy as a predictor of catalepsy in rats: a pharmacokinetic-pharmacodynamic modeling approach. Pharm. Res. 31, 2605-2617 (2014).

75. Agarwal, N., Port, J.D., Bazzocchi, M. \& Renshaw, P.F. Update on the use of MR for assessment and diagnosis of psychiatric diseases. Radiology 255, 23-41 (2010).

76. Mason, G.F. \& Krystal, J.H. MR spectroscopy: its potential role for drug development for the treatment of psychiatric diseases. NMR Biomed. 19, 690-701 (2006).

77. Borsook, D., Becerra, L. \& Hargreaves, R. A role for $\mathrm{fMRI}$ in optimizing CNS drug development. Nat. Rev. Drug Discov. 5, 411-424 (2006).

78. Borsook, D., Becerra, L. \& Fava, M. Use of functional imaging across clinical phases in CNS drug development. Transl. Psychiatry 3, e282 (2013).

79. Prichep, L.S. Use of normative databases and statistical methods in demonstrating clinical utility of QEEG: importance and cautions. Clin. EEG Neurosci. 36, 82-87 (2005).

80. Prichep, L.S., John, E.R., Howard, B., Merkin, H. \& Hiesiger, E.M. Evaluation of the pain matrix using EEG source localization: a feasibility study. Pain Med. 12, 1241-1248 (2011).

81. Alhaj, H., Wisniewski, G. \& McAllister-Williams, R.H. The use of the EEG in measuring therapeutic drug action: focus on depression and antidepressants. J. Psychopharmacol. 25, 1175-1191 (2011).

82. Zuideveld, K.P., Van der Graaf, P.H., Peletier, L.A. \& Danhof, M. Allometric scaling of pharmacodynamic responses: application to 5Ht1A receptor mediated responses from rat to man. Pharm. Res. 24, 2031-2039 (2007).

83. Yassen, A., Olofsen, E., Kan, J., Dahan, A. \& Danhof, M. Animal-tohuman extrapolation of the pharmacokinetic and pharmacodynamic properties of buprenorphine. Clin. Pharmacokinet. 46, 433-447 (2007).

84. Reese, T.S. \& Karnovsky, M.J. Fine structural localization of a bloodbrain barrier to exogenous peroxidase. J. Cell Biol. 34, 207-217 (1967). 